
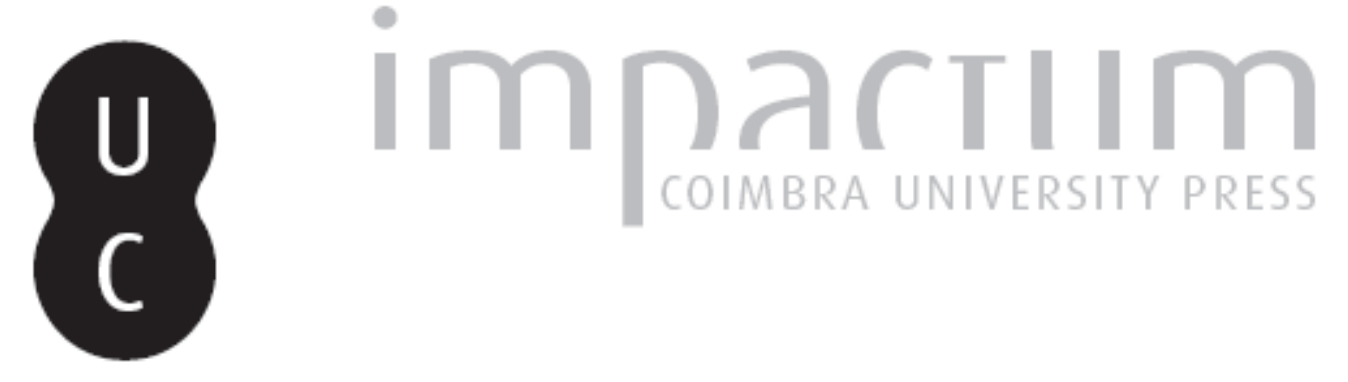

\title{
Modelli di ermeneutica platonica nel secoli XIX e XX
}

Autor(es): Rowe, Christopher

Publicado por: Imprensa da Universidade de Coimbra

URL persistente:

URl:http://hdl.handle.net/10316.2/42281

DOI:

DOI:https://doi.org/10.14195/2183-4105_1_4

Accessed : $\quad$ 26-Apr-2023 15:25:16

A navegação consulta e descarregamento dos títulos inseridos nas Bibliotecas Digitais UC Digitalis, UC Pombalina e UC Impactum, pressupõem a aceitação plena e sem reservas dos Termos e Condições de Uso destas Bibliotecas Digitais, disponíveis em https://digitalis.uc.pt/pt-pt/termos.

Conforme exposto nos referidos Termos e Condições de Uso, o descarregamento de títulos de acesso restrito requer uma licença válida de autorização devendo o utilizador aceder ao(s) documento(s) a partir de um endereço de IP da instituição detentora da supramencionada licença.

Ao utilizador é apenas permitido o descarregamento para uso pessoal, pelo que o emprego do(s) título(s) descarregado(s) para outro fim, designadamente comercial, carece de autorização do respetivo autor ou editor da obra.

Na medida em que todas as obras da UC Digitalis se encontram protegidas pelo Código do Direito de Autor e Direitos Conexos e demais legislação aplicável, toda a cópia, parcial ou total, deste documento, nos casos em que é legalmente admitida, deverá conter ou fazer-se acompanhar por este aviso.

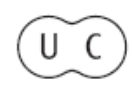




\section{Modelli di ermeneutica platonica nel secoli XIX e XX}

ROWE, Christopher, in 1. Plato 1 (2001),

[En ligne], January 2001

Questo contributo e basato su una lezione fatta all' Università di Macerata. Ho appena finito di tenere la mia lezione alla classe di Maurizio Migliori sul Fedro, dando un resoconto del mio metodo di lavoro che (penso) di aver impiegato nell' analizzare i testi di Platone. Il mio resoconto è iniziato con:

"La prima e dal mio punto di vista più rilevante caratteristica del corpus - caratteristica che io credo dobbiamo sempre tenere in mente - é che esso é uno strano insieme di scrittura. Altri filosofi scrivono oscuramente, sia che lo vogliano o meno; la differenza con Platone è che anche la forma in cui egli scrive richiede un'interpretazione. A grandi linee noi sappiamo come rispondere a un trattato filosofico (seriamente, e riflettendo) o a una pièce teatrale (piangiamo, abbiamo paura, ridiamo) o ad ogni altro riconosciuto genere letterario: anche se lo scrittore tenta di impressionarci ingannando le nostre attese, tuttavia in un certo qual modo capiamo cosa sta accadendo. Ma non c'è nulla che corrisponda nella forma a un dialogo platonico. A volte la conversazione viene riportata da altri, altre volte é tutta in discorso diretto; occasionalmente ci sembra di capitare in un dialogo in pieno svolgimento. L'autore, Platone, è permanentemente assente come tale e solamente una volta o due abbiamo un riferimento a lui, presente o assente. Se i dialoghi sono più di ogni altra cosa simili a una pièce, tuttavia non sono delle pièce: non vengono rappresentate in teatro né di fronte a un uditorio (se una volta lo sono stati, sono stati letti da un'unica voce), e sono spesso piuttosto non-drammatici - non accade molto e anche quando qualcosa accade, al lettore /ascoltatore è richiesto un duro lavoro affinché possa capire che cosa sta accadendo.E' 1'intensità di quello che uno potrebbe chiamare il contenuto intellettuale dei dialoghi platonici, e il modo nel quale un tale contenuto è stato ordito in profonde strutture, tutto questo conferisce in primo luogo a loro la loro stranezza. Questo chiaramente fa sorgere la domanda sul perché Platone avrebbe scritto in questa maniera: perché egli ha bisogno di fare lavorare i suoi lettori?"

Questo mi ha sembrato un punto importante da sottolineare: Platone è difficile e ha bisogno di essere interpretato. Ma questo è un punto controverso in un certo qual modo, in quanto c'è di più in Platone di quello che sembra a prima vista, più di una collezione di testi variegati, coloriti e deliziosamente differenti, sui quali noi potremmo lavorare e gustare individualmente. Suppongo che ci possa essere un parallelo con un autore come Luciano, con la differenza che Platone è più complesso è più sofisticato. Infatti Platone è sotto molti aspetti uno scrittore diverso di Luciano. Ma la possibilità del paragone risiede nell'idea che come uno non ritiene di dover scavare a fondo per trovare il significato in Luciano, così forse il significato di Platone è anch'esso più vicino alla superficie di quanto comunemente supponiamo. Le difficoltà da questo punto di vista sorgono dal tentativo di individuare alcuni tipi di unità nelle numerose caratteristiche del lavoro di Platone. A grandi linee questa sembra essere stata 1'idea di George Grote nei suoi tre volumi intitolati "Plato and the other companions of Socrates" (London 1865); quest'idea inoltre riflette le pratiche di lavoro di molti interpreti di Platone che potremmo chiamare "letterari", un nuovo movimento che no ha nessuno reale riscontro nello studio platonico dell'antichità o del XIX secolo, per non dire della prima parte del ventesimo secolo. Ci sono stati tuttavia altri studiosi come ad esempio il grande Wilamowitz i quali in maniera simile hanno sottovalutato la filosofia in Platone, benché per ragioni differenti.

Le cause di questo nuovo movimento risiedono in quella che potrebbe essere una reazione moderna all'appropriazione di Platone da parte dei filosofi. Questa reazione è stata incoraggiata dalla crescita dei dipartimenti di letteratura comparativa specialmente negli USA; è stata inoltre incoraggiata da una ancora più estrema forma di appropriazione di quella che a grandi linee potrebbe essere definita la tendenza analitica o anglosassone in filosofia. Questa tradizione nella forma più estrema è capace di sezionare i lavori platonici seguendo le loro argomentazioni, formalizzandole e specie mostrando le loro fallacie e il modo per risolverle (i filosofi di Oxford in particolare amano pensare a questa come la tendenza di una scuola di Oxford). Nelle ultime due decadi siamo cresciuti ascoltando la pretesa secondo la quale dobbiamo prestare attenzione non solo alle argomentazioni di Platone ma anche alla forma in cui egli a scelto di esprimere le sue idee (se lo ha fatto), cioè agli aspetti letterari dei dialoghi, un punto di vista perfettamente legittimo incoraggiato per differenti ragioni da Leo Strauss e dei suoi seguaci. Nella forma più estrema di questa tendenza letteraria ci sono state effettivamente versioni di alcuni dialoghi prodotti per il palcoscenico che hanno semplicemente tralasciato gli argomenti importanti (non intendo dire molto di più sugli Straussiani, i seguaci o gli epigoni di Leo Strauss, i quali hanno sviluppato la loro 
interpretazione di Platone su quello che più tardi chiainerò il Platone esoterico o esoteristico, prendendo prestito la terminologia da Tigerstedt. Questo Platone soprattutto esprime punti di vista politicamente sani in modo che essi siano compresi da lettori "gentlemen", mentre non dice nulla che possa disturbare gli intelletti meno acuti delle masse. Questa è una scuola di interpretazione che normalmente finisce suggerendo che i dialoghi, se correttamente interpretati, possano significare 1'opposto di quello che sembrano significare a prima vista. Deve essere ricordato che al pari di molte scuole interpretative, alcuni Straussiani di gran lunga più sofisticati di altri e che loro scritti possono essere letti con profitto anche se non vorrei entrare nella questione se questo possa essere attribuito alle qualità dei singoli interpreti, come ad esempio Jacob Howland, più che allo Straussianesimo come tale.)

Infatti il paragone che ho appena fatto tra interpreti letterari e correnti di Platone e George Grote è probabilmente assai ingiusta nei confronti di Grote. Le sue analisi di singoli dialoghi sono frequentemente più che degne di essere letti: egli ha un buon occhio per gli argomenti in Platone, occhio che è stato raramente eguagliato in quanto ad acutezza. Le sue controparte moderne, per contrasto, sono, come ho suggerito, raramente interessati alle argomentazioni di Platone, preferendo usare i suoi testi come un campo nuovo e congeniale all'esercizio di particolari teorie o approcci ermeneutici (alcuni di questi innominati interpreti sono influenzati da Derrida, sul cui approccio del Fedro 1'ultima parola, o comunque 1'ultima parola che bisogna dire, è stata pronunciata dal mio collega di Durham, Seàn Burke, nella seconda edizione del suo libro "The Death and Return of the Author", Edinburgh UP, 1998). In ogni caso Grote ha qualcosa da dirci su Platone che questi interpreti più recenti spesso no hanno. Il mio punto, tuttavia, nell'introdurre Grote e gli altri era rendere evidente che è possibile non trattare Platone come problematico, come io ho suggerito all'inizio dovrebbe essere trattato. A questo io sono tentato di replicare: che assumere un punto di vista come quello di Grote significa già interpretare Platone e che questa, come ogni altra interpretazione, $\mathrm{o}$ avrà successo o sarà sconfitta dall'evidenza. $\mathrm{E}$ a me personalmente essa sembra immediatamente sconfitta quando uno inizia a riflettere sul fatto che i dialoghi, benché siano indipendenti formalmente 1'uno dall'altro, infatti sembrano condividere le stesse preoccupazioni. Esaminate il tema più ricorrente, tema continuamente enfatizzato, $\mathrm{e}$ accettato, dai personaggi nei dialoghi (spesso anche rigettato da altri personaggi che tuttavia non hanno 1'ultima parola):

1 'importanza, cioè, di fare filosofia come attività opposta a qualsiasi altra. Ora è impossibile che un autore abbia scelto di far trattare ai suoi personaggi un tema senza avere egli stesso alcuna interesse personale in esso, ma sembra scarsamente economico di supporlo (e qui mi dichiaro un ardente sostenitore di Ockham). Io stesso considero ciò tanto sicuro quanto qualsiasi cosa può essere nel caso di un autore che ha risolutamente rifiutato di dirci qualcosa direttamente su se stesso, cioè che Platone stesso ha ritenuto la filosofia la più importante attività al mondo, e che egli ha scritto in parte per convincere gli altri a pensare la stessa cosa. Ma questo rilievo in se stesso dà già alla sua scrittura una certa unità, almeno di scopo.

Un'altra importante conseguenza è che Platone è capace di esprimersi indirettamente: c'è di più in lui di quello che sembra a prima vista, come ho appena detto, di più di quella inevitabile relazione, spesso basata sulla collusione, tra autore e lettore. I dialoghi sembrano essere una cosa sola (conversazioni oppure registrazioni di conversazioni), mentre effettivamente essi sono qualcosa d'altro (anche?), cioè un invito alla filosofia. Ma chiaramente ci sono molte altre idee e temi comuni che scorrono nelle differenti parti del corpus platonico. E' pertanto dal mio punto di vista inevitabile supporre, e forse sbagliato il non farlo, che Platone esprima se stesso tipicamente, in forma indiretta: mentre ci offre quella che sembra essere una collezione di lavori più o meno indipendenti (di qualsiasi tipo essi siano), egli infatti si sta anche offrendo una collezione di lavori che hanno connessioni implicite ma profonde fra di loro.

Ma chiaramente è qui che il problema inizia: abbiamo bisogno di una spiegazione sul perché Platone abbia lasciato queste connessioni meramente implicite (perché egli si nasconde così completamente dietro ai suoi personaggi?); abbiamo anche bisogno di una spiegazione circa le differenze tra i dialoghi, perché, come tutti sanno, quando ricorrono gli stessi temi essi lo fanno in nuove forme e non infrequentemente in modo da sembrar contraddire quello che è stato detto su di loro in questo dialogo o in altri. L'approccio di Grote evitava entrambe questa necessità: Platone era in effetti lì, sulle spalle del lettore il quale poteva semplicemente deliziarsi nel seguire gli itinerari contorti di una mente sofisticata, senza troppo occuparsi di quello che poteva scorrere più in profondità. Sembra tuttavia che ora noi dobbiamo andare più in profondità e il problema ora riguarda 1 'indeterminatezza dei segnali per noi che siamo così lasciati senza alcuna certezza circa la forma generale di quello che stiamo cercando.

Questo è quello che uno dovrebbe inevitabilmente concludere seguendo una prospettiva storica e così c'èstato poco accordo tra gli interpreti, almeno negli ultimi due secoli. (Né sembra che ci possa essere consenso su ciò nel prossimo secolo: c'è probabilmente meno accordo ora su come leggere Platone di quanto ce ne sia stato negli ultimi duemila cinque cento anni, o almeno questa è la mia impressione sulla base di articoli e libri che hanno occupato la mia scrivania nell'ultimo decennio, prima nel mio ruolo di recensore per il periodico "Phronesis" e poi come direttore della rivista.)

Prima del XIX secolo la scena era dominata dagli interpreti neoplatonici, i quali potevano a grande linee pretendere di discendere direttamente dai successori di Platone nell'Accademia; tale ereditarietà è stata seriamente minacciata solo una volta, benché in maniera significativa, dagli scettici della Nuova Accademia, essi stessi pensando di essere i veri eredi. Infatti oggi simili battaglie vengono combattute tra i dogmatisti, cioè coloro che pensano come i neoplatonici che Platone sia importante principalmente per un certo tipo di sistema filosofico, e gli scettici, che invece vedono Platone come in continua ricerca, come forse Socrate nel Simposio. Non sembra esserci tuttavia una facile decisione tra le due schiere. Tuttavia penso che siano in pochi coloro che accetterebbero un metodo neoplatonico di leggere Platone: la relativamente recente crescita negli studi neoplatonici non dipende dall'illuminazione che personaggi come Plotino possono portare alla comprensione del corpus platonico, ma dall'intrinseco valore dei neoplatonici stessi 
come filosofi con tutti i loro diritti. Bisogna qui procedere con attenzione perché esiste un numero significativo di persone che ritiene che la filosofia di Platone sia più vicina a quella dei neoplatonici (quegli ultra-dogmatisti) che a quella degli scettici. La mia idea è solamente che pochi oggi leggerebbero i dialoghi come i neoplatonici, cioè come testimonianze di metafisica neoplatonica (un compito che ha richiesto e richiederebbe non solo ingenuità ma una credenza indipendente e completa ai punti di vista metafisici in questione). Se questo tipo di Platone deve essere trovato da qualche parte, è solo sporadicamente nei testi che abbiamo: e questo è uno dei pochi punti su cui tutti gli studiosi di Platone oggigiorno concordano.

Il dominio dell'interpretazione neoplatonica non ha significato che nessuno abbia letto Platone di per se stesso; alcuni lo hanno fatto chiaramente (e uno pensa ai platonisti del Rinascimento fiorentino, o a Edmund Spenser e ai Platonisti di Cambridge, specialmente Ralph Cudworth, in Inghilterra). Ciò nondimeno, fu solo con il declino e la caduta della interpretazione neoplatonica (alludo chiaramente al titolo della monografia di Tigerstedt; "The Decline and Fall of the Neoplatonic Interpretation of Plato" (Commentationes Humanarum Litterarum 52, Helsinki, 1974) che quella che oggi è riconosciuta come la moderna critica di Platone ha iniziato ad emergere. L'evento più importante in questo processo iniziato con questa rottura è la pubblicazione della traduzione di Platone da parte di Schleiermacher negli anni dal 1804 in avanti, pubblicazione che ha inteso il sistema di Platone ricostruibile dai dialoghi stessi, un punto di vista che da allora è diventato più o meno standard. Che Platone avesse un sistema era dato per scontato, seguendo Schleiermacher, Platone lo aveva completamente formato fin dall'inizio, esponendolo tuttavia gradualmente iniziando col Fedro. Egli dice "'the depreciation of writing in comparison with true and living philosophical communication [i.e. in the Phaedrus 1 ... itself perfectly intelligible as a justification of Socrates' abstinence from writing, and as a sentiment inspired by that method of teaching which Plato at that time despaired of ever imitating in written treatises, though he afterwards learnt to do so ... But perhaps that author [Tennemann, whom Schleiermacher is in the process of criticizing] does in reality hold to another ground still behind that already brought forward; namely that in the Phaedrus so much that is Platonic appears, while he is only disposed to consider those writings of an early date which connect themselves immediately with Socrates, and in which the peculiar style of Plato is still wanting, esteeming so large a work and with such a subject as only adapted to later times. But every skilful and self-experienced person will certainly allow that true philosophizing does not commence with any particular point, but with a breathing of the whole, and that the personal character of the writer, as well as the peculiarities of his modes of thought and views of things in general, must be to be found in the first commencement of the really free and independent expression of his sentiments. Why, therefore, should not the communication of the Platonic philosophy begin thus?' (cito dal "Schleiermacher's Introductions to the Dialogues of Plato"; tradotto dal tedesco da W. Dobson, Cambridge 1836; ristampato da Arno Press, 1973, p.60). Qualcosa su cui vorrei parlare qui ma su cui non mi sento sufficientemente qualificato è 1'influenza su Schleiermacher da parte del Romanticismo, specialmente di quello di Friedrich Schlegel, suo amico.

E' l'idea di sistema attorno alla quale può venire organizzata convenientemente la storia delle interpretazioni platoniche del XIX e XX secolo, benché ammetta che in questo caso verrebbe fatta ingiustizia alle varie tradizioni di pensiero, sia che (a) gli interpreti ritengano che Platone abbia un sistema sia che (b) essi leggano Platone senza attribuirgli alcun sistema.

Membri del primo gruppo devono affrontare il problema della caratteristica dei dialoghi con cui ho iniziato, benché non tutti riconoscano questo problema: che i dialoghi non sembrano ovviamente rappresentare un'unità sistematica. Per salvare quindi la loro ipotesi originale, ciò che Platone sia sistematico, sono obbligati ad adottare uno o più strategie possibili: devono offrire un resoconto della filosofia platonica piuttosto innocuo (come quello di Shorey, forse 1'ultra-unitario), oppure devono eliminare parti che non si adattano alla loto interpretazione, dichiarandole spurie (caratteristica questa dell'interpretazione del XIX secolo, specie dei tedeschi: adesso c'è in alcune parti del mondo un egualmente estrema ma di segno opposto reazione, che consente a malapena la possibilità dell'esistenza di parti spurie in un corpus tradizionale); oppure devono spiegare le differenze e la contraddizioni in termini di diverse tesi genetiche, riferendosi alle connessioni tra biografia, la psicologia, e 1'evoluzione del pensiero di Platone (cioè in modi differenti Hermann, Wilamowitz, e altri, specialmente in XX secolo, basandosi, or pensandosi basati, ai risultati aggiunti dagli stilometrici: ci tornerò più tardi sul tema). Alternativamente, coloro che intendono scoprire un sistema in Platone lo hanno fatto scoprendolo al di fuori dei dialoghi pubblicati (pariando cioè di "agrapha dogmata", o più generalmente basandosi su quanto accadeva nell'Accademia: mi riferisco qui soprattutto a quella che è conosciuta come la scuola "tubinga-milano", benché questo tipo di approccio abbia avuto molti seguaci prima dell'arrivo sulla scena dei tubinghesi Gaiser e Kramer).

Basta per quanto riguarda il primo gruppo, cioè quelli che partono con 1'assunto che Platone abbia un sistema, cosa che si pensano di poter dimostrare. Questo gruppo poi si rivela essere piuttosto ampio, includendo studiosi cresciuti all'interno di tradizioni filosofiche piuttosto differenti. Qualcuno potrebbe chiedersi se coloro che accettano la versione di chi vede Platone e il suo pensiero divisibile in un prima, un poi, e un dopo dovrebbero essere classificati con i sistematici. In effetti questo modello, quello della tesi genetica, implica 1'attribuzione di un sistema piuttosto ben organizzato al Platone di mezzo, anche se poi tale attribuzione potrebbe significare un abbandono del sistema da parte dell'ultimo Platone, anche se rimane vero che chiunque abbraccia quest'ultima interpretazione deve, in un certo qual modo, appartenere al gruppo dei non-sistematici. Membri di questo secondo gruppo, che non credono affatto in un sistema platonico, da un lato includono gli "atomizzanti "come Grote, e gli interpreti letterari, e dall'altro tutti coloro che generalmente rinunciano alla fede in un sistema, ritenendo, diversamente dai membri del primo gruppo, che questo non è ciò su cui verte la filosofia ma come gli altri ritenendo che Platone dopotutto sia un filosofo (cosa che, abbiamo visto, non è un assunto generalmente condiviso). (E' qui nella disputa moderna tra sistematizzanti e anti-sistematizzanti che noi troviamo il proseguo della 
disputa antica tra interpreti scettici e dogmatici a cui mi sono riferito poco fa.) E' mia impressione che quest'ultimo gruppo, gli antisistematizzanti, stia crescendo in influenza, in particolar modo perché il modello dominante della tesi genetica viene continuamente messo in discussione (pur rimanendo dominante per il momento), e anche a causa della rinnovala enfasi sull'importanza di considerare Platone insieme al suo messaggio (per alludere a Marshall McLuhan). Se uno riflette attentamente sul modo in cui Platone sembra distanziare se stesso dai suoi testi e sul modo in cui qualsiasi conclusione positiva dei dialoghi tenda ad essere provvisoria, allora la tentazione di tornare all'immagine di Platone come uno scettico qualificato è probabilmente piuttosto fascinante, specialmente se uno ha una certa antipatia per le costruzioni sistematiche (quando io stesso penso "Platone ha pensato ... ", un'immagine mi fluttua davanti di un autore che dice "Che cosa? Io? Non c'ero mai, e solamente perché i miei personaggi hanno raggiunto quella conclusione in quell'occasione, perché voi ritenete che io pensi sia una buona conclusione, specie se ho fatto continuamente ribadire a loro i limiti della prospettiva umana?").

Qui voi avete un riassunto generale del mio argomento; "Modelli ...". Il mio compito è stato reso più semplice dall'esistenza di un libro, per certi versi ammirabile, di Tigerstedt, titolo "Interpreting Plato" (Acta Univ. Stockholmiensis, Stockholm Studies in History of Literature 17, Stockholm, 1977). Mi sono prima riferito ad un altro libro di Tigerstedt, "Decline ... ", e probabilmente egli conosce come nessun altro 1 'intero esercito di Platoni che la storia ci presenta. Il mio precedente resoconto, come immediatamente riconosco, e come sarà stato chiaro in ogni caso, ha un considerevole debito nei confronti del suo, benché il suo principio di organizzazione sia leggermente differente, e in più il libro stato scritto nel 1977, prima dell'emergenza delle nuove tendenze interpretative che ho menzionate (il campo degli studi platonici sembra a volte assumere un aspetto quasi-eracliteo: dove sono i punti fissi?). Tigerstedt inizia con "II Problema", cioè dell'interpretazione platonica: come capire questa figura apparentemente così camaleontica? (O è questo piuttosto un non-problema, come Grote ha proposto in effetti?) Tigerstedt poi si sposta sugli "athetizers" ("The Resort of the Scalpel ('scalpello'): 3 pagine), su alcuni critici "Whig" di Platone che spiegano tutto in termini di progresso (3 pagine), sul "Genetic Approach" (27 pagine), sugli unitari (Shorey, Hofmann, Robin, Comperz: 11 pagine), sugli approcci esoterici ("The Hidden System". 19 pagine), e infine sul suo approccio preferito (17 pagine). Tutto questo ha perfettamente senso, fatta eccezione per una o due cose. In primo luogo, lo schema di Tigerstedt piuttosto stranamente tratta quelli che potrebbero essere chiamati i "patronisers" di Platone, che includono ma che non sono co-estensivi con la scuola interpretativa di "Oxford", come in grado di risolvere il problema platonico che ha appena identificato (citazione: "contradictions, gaps; obscurities, and ambiguities ...", p. 22), quando infatti essi stanno semplicemente applicando lo stesso metodo che applicano nel trattare 1'intera storia della filosofia: 1'assunto è che le cose vanno sempre migliorando (a volte possono andare all'indietro, com'è accaduto dopo Aristotele). Una caratteristica particolare di questo approccio, caratteristica che nella mia esperienza io associo agli studiosi educati a Oxford, è la credenza che Platone abbia fatto degli errori (o sollevato i problemi) mentre Aristotele li ha risolti (un'altra variante di questo tipo di approccio è quella condivisa da coloro che considerano la storia della filosofia antica come premessa alla rivelazione cristiana). L'altra e più importante obiezione che devo fare al libro di Tigerstedt ha a che fare con la quantità di spazio che egli riserva a demolire 1'interpretazione esoterica: non è che io sia in disaccordo con 1'obiezione che egli fa all'esoterismo (o esotericismo, come preferisco) in tutte le sue forme, ma perché questa critica sbilancia il libro. La verità è che gli esotericisti sono animali più rari di alcuni specie di geneticisti, nonostante le paure di Tigerstedt (citazione: "although the Esoterists ... have encountered sharp criticism, they have also won eager approval"; p. 63, anche da parte del sobrio Albin Lesky), e il fatto che gli argomenti anche contro i più sofisticati geneticisti sono, dal mio punto di vista, altrettanto forti (tornerò su questo aspetto più tardi).

Tuttavia, ritengo che sia difficile scrivere una storia su un soggetto che ti appartiene in un certo qual modo senza tradire le proprie affiliazioni; ed è certamente importante per noi, come interpreti, avere una prospettiva storica sulle nostre stesse posizioni. E' sempre umiliante scoprire quanto frequentemente uno si riduca a ripetere vecchi argomenti e a combattere entusiasticamente battaglie già combattute molte volte nel passato, benché si debba dire che molti interpreti contemporanei sembrano preferire un lavoro entro $\mathrm{i}$ confini di uno certo paradigma, senza un chiaro senso di continuità o discontinuità con il passato. Se mi venisse chiesta la mia posizione all'interno dello spettro interpretativo che la storia ha mostrato essere se non possibile, almeno concepibile, sono tentato di citare un passo di Luigi Stefanini, parzialmente citato da Tigerstedt stesso:

"Una certezza assoluta e un dubbio radicale, un oggetto fisso e immutabile proposto alla conquista dello spirito, è un soggetto ansiosamente proteso incontro ad esso e sempre respinto dalla meta; un' intuizione che istantaneamente e prodigiosamente si eleva in alto, e un dialettica faticosa che non riesce mai a imprigionare la subitanea rivelazione e pur è necessario presupposto e conseguenza di questa: ecco 1'atteggiamento essenziale del pensiero platonico. - Non esiste nella storia della filosofia un termine che valga a esprimere con precisione 1'atteggiamento stesso ... ['Probabilismo' non è adatto, nonostante I'Accademia Nuova:] 'Se parlassi di probabilismo, indicherei sì 1 ' incapacità della mente umana di porsi in equazione perfetta con la verità, ma non terrei conto degli altri mezzi con cui il platonismo garantisce la certezza [?]. Se parlassi di misticismo, darei ragione dello slancio affettivo con cui 1' anima si protende verso il suo compimento, ma trascurerei 1' aspetto razionalistico e quasi illuministico del sistema per cui 1' uso del raziocinio è 1' antecedente non eliminabile di ogni progresso dello spirito. Costretto a servirmi d'un termine, pur inadeguato, che rappresenti il massimo grado di approssimazione, parlo di scepsi platonica, intendendo la parola nel significato etimologico di ricerca. Platone è il ricercatore instancabile di ciò che ha già trovato; per meglio dire, lo sforzo della sua speculazione è teso a tradurre in termini razionali ciò che 1' intuito ineffabilmente gli ha rivelato. Giunto al limite della ricerca, egli non dichiara inconoscibile, ma semplicemente non conosciuto da lui ciò che sta al di là; non dubita della verità, ma delle prove della verità" 
(Platone, 1, Padova 1949 [seconda ed., ristampata nel 1991], p. xxxiii).

Questo copre molti aspetti del mio punto di vista e così pienamente che è un peccato che io debba lasciare indietro Stefanini, specialmente quando egli si aggiunge a quelli che collegano la conoscenza di cui Platone andava in cerca ma che non riusciva a trovare con la verità cercata e pretesamente posseduta dalla Chiesa (Stefanini, Platone, II, pp. 45-6). In un certo qual modo anche qui Stefanini sicuramente individua i punti centrali del pensiero platonico meglio di quanto facciano alcuni interpreti moderni, i quali tendono a sottostimare l'adesione di Platone a certe verità, almeno quelle di un tipo generale, di cui una, penso, dovrebbe essere chiamata "platonism", per quanto la si prenda riduttivamente. Vorrei cioè dire che non trovo alcuna difficoltà nell'affermare che Platone era un Platonista, nel senso che credeva fermamente che esistessero cose là fuori da conoscersi.

Questo è un aspetto che è stato negato nel secolo scorso. Mi riferisco a H.-G. Gadamer, il quale, per citare da un contributo di Christopher Gill offerto in un recente colloquio su Platone e Socrate, 'has found in Platonic dialogue an antecedent of his hermeneutic view that truth arises out of the dialectical sharing of interpretations, formed from an inevitably localized standpoint', cosa che ha portato Gadamer a dichiarare che "Platon war kein Platoniker". Un punto di visto similare ma più moderato può essere identificato in Donald Davidson, il quale ha inteso il metodo socratico di dialettica nel Filebo come nei dialoghi giovanili, "come anticipante la sua stessa concezione intersoggettiva su quello che viene coinvolto nello stabilire la verità" (cito Gill ancora, J. Annas and C. Rowe, eds., Perspectives on Plato: Modern and Ancient, Cambridge, Mass., forthcoming). Qualsiasi difficultà che noi potremmo avere con una nozione oggettiva di verità, veramente con 1'apparente adesione di Platone alla nozione di dialogo, è sicuramente più duro non trovare un'altrettanto ossessiva preoccupazione nei dialoghi per la scoperta della verità che è tanto fuori di noi e indipendente di qualsiasi accordo o disaccordo tra gli uomini quanto lo è ciaseun mondo concepito 1à fuori.

Questo aspetto deve presupporre che sia effettivamente possibile raggiungere conclusioni sui testi di Platone e in generale su tutti testi. Questo mi porta indietro agli sviluppi moderni a cui mi sono riferito prima. Sviluppi nella teoria letterale moderna hanno avuto inevitabilmente un impatto sugli studi platonici, nella stessa maniera in cui lo hanno avuto lo sviluppo della teoria psicanalitica alla fine del XIX secolo e all'inizio del XX secolo, e certi movimenti o teorie politiche. A grandi linee sono contento di dover trattare tali sviluppi nella stessa maniera: cioè salvando e se necessario assimilando qualsiasi cosa che possa essere suggerita da una particolare teoria, e poi tornando arricchito, frustrato o infuriato ai testi di Platone, i quali di per se stessi sollevano sufficienti problemi, molti dei sovrapponentisi con quelli posti dai teoristi, come per esempio con quelli dei deconstruzionisti o dei teoristi del "reader-response". Questo privilegiare i testi implica sicuramente un'adesione alla vecchia idea che è possibile, almeno in principio, decidere problemi interpretativi o almeno scegliere la migliore interpretazione disponibile; e se non siamo in grado di raggiungere una tale decisione, questo è imputabile alla mancanza di una chiara evidenza (che nel caso di Platone non esclude quello che dalia prospettiva dell'interprete potrebbe sembrare deliberatamente scherzoso da parte dell'autore). Questo non è un punto, o non solo un punto, di eccessiva confidenza in un progetto illuminista, o un tipo di Platonismo già in pensione; piuttosto è un punto di adesione simpatetica a quello che io trovo assolutamente fondamentale in Platone, la fede cioè nella possibilità di argomentare (una fede che tuttavia che è abbastanza aperta da riconoscere i suoi limiti). Alcune forme di relativismo possono apparire piuttosto di moda, ma nel caso dei scritti filosofici, anche uno come Platone (se ci fosse un altro come lui), ciò mi sembra irrilevante. D'altra parte devono ancora convincermi che esiste qualcosa nei dialoghi che rende necessario per noi supporre che Platone abbia qualcosa degno di essere descritto come un sistema, almeno per quanto ciò implichi un paragone con i grandi costruttori di sistemi dell'idealismo tedesco.

Basta con la mia posizione, che penso sarebbe largamente condivisa nei suoi aspetti antirelativistici, se non in altri (questo particolare modello non è privato). Vorrei ora finire parlando brevemente di due moderni sviluppi, uno piuttosto recente, 1'altro con le sue radici nel passato. Negli ultimi due decenni è emerso un campo specialistico, nel suo senso stretto esistente solo nei USA, e spesso chiamato "Socratic studies". Esso consiste (ancora, definendolo strettamente) nel tentativa di arrivare ai punti di vista del Socrate storico facendo affidamento, a parte qualche occasionale riferimento a Senofonte e Aristotele, su quelli che sono chiamati i dialoghi giovanili o socratici di Platone. L'esponente più illustre di tale tendenza il defunto grande Gregory Vlastos, i cui punti di vista sulla materia sono riassunti nel suo "Socrates: Ironist and Moral Philosopher", 1991. Ora la storia delle interpretazioni di Socrate è un argomento di per se stesso; qui però mi limiterò a dire che il tipo di approccio in questions è ovviamente dipendente da alcuni tipi di interpretazione genetica di Platone; più specificamente da tipi di geneticismo, adesso molto comuni, che identificano il primo Platone con un periodo in cui lui era socratico, da cui il titolo di "dialoghi socratici", i quali ci forniscono più o meno completamente 1 'autentica dottrina del vero Socrate. Questo punto di vista ha le sue radici nel primo diciannovesimo secolo o addirittura prima (forse nel Romanticismo: vedi Alexander Nehamas, "The Art of Living". (University of California Press, 1977) 1998, pp. 92 ff), e riflette la tendenza a ricostruire un Socrate differente da quelli che i secoli precedenti hanno ricostruito, specialmente diverso dai vari Socrate che troviamo nella tradizione filosofica del quarto secolo a.C. e più tardi. Rimane da esaminare la possibile durata di questo tipo di "Socratic studies"; ci sono certamente altri tipi che sembrano meritare di durare più lungamente. Una questione speciale o ignorata o sottovalutata da questi interpreti riguarda la ragione per cui 1'evidenza di Platone su Socrate dovrebbe essere preferita a quelia di qualsiasi altro. Ma proprio 1'idea di un periodo socratico in Platone, un'idea che per quanto ne so non è mai stata universalmente accettata, e che è stata virtualmente inconcepibile prima dell'inizio del XIX secolo, è ora costantemente attaccata, insieme all'interpretazione genetica o "developmentalist" di Platone.

Qui mi accontento di menzionare un unico antigenetista, Charles Kahn. Il suo nuovo libro, "Plato and the Socratic Dialogue" (Cambridge, 1996) non sta esercitando un'influenza significativa, in parte forse a causa della natura molto specialistica della sua tesi 
principale, che in effetti tratta una dozzina di dialoghi "giovanili" e "medi" come parti di un singolo progetto che vede la Repubblica come suo culmine. Ma i argomenti di Kahn rivolti contro il modello geneticista classico devono essere secondo me ascoltati. E infatti in uno aspetto importante, i argomenti sono inattaccabili. Sto qui pensando a un nuovo lavoro di Kahn (che parerà nella stessa collana del saggio di Christopher Gill che io ho citato prima, Perspectives on Plato), in cui egli sottolinea che la usuale divisione dei dialoghi in "giovanili", "medi", e "tardi" non corrisponde con la divisione dei dialoghi in tre gruppi fatta degli stilometristi. Se allora conferiamo credito alle conclusioni degli stilometristi, dobbiamo effettivamente lasciarcele dietro per poter continuare a parlare tradizionalmente di dialoghi come "medi", distinti cioè dai "giovanili" e dai "tardi" (così il Fedro e Simposio appartengono al primo gruppo secondo gli stilometristi, mentre due dialoghi del loro secondo gruppo vengono considerati "tardi". Per quelli di noi cresciuti nella tradizione geneticista, questa non è meno che una rivelazione, o dovrebbe esserlo, dal momento che ci è sempre stato detto (c mi dispiace di averlo preso in fiducia) che parlando in grandi linee, gli studi stilometrici scientifici (?) hanno supportato le intuizioni dei geneticisti. Sembra quindi che se voglianio rimanere un tradizionalista (cioè uno che crede che i dialoghi possano dividersi in "giovanili". "medi", "tardi"), dobbiamo preferire gli argomenti "filosofici" per la datazione dei dialoghi piuttosto che quelli stilistici. Una via d'uscita sarebbe quella di negare la validità o la forza delle argomentazioni stilistiche; effettivamente dal mio punto di vista sono gli argomenti filosofici che sono al meno in molti casi i più deboli.

Qui debbo finire il mia contributo, con quello che potrebbe essere un punto di svolta negli studi platonici, e una significativa rottura nel modello dominante. Il mio è stato per certi versi un resoconto affrettato dell'argomento: ci sono state alcune importanti omissioni (non ho detto nulla della scuola di Marburgo), e alcuni commenti su alcune figure centrali sono state talmente brevi da risultare caricaturali. Spero tuttavia di aver fornito un certo modello, anche se sbilenco, per capire i più importanti, o almeno i più diffusi, "modelli di ermeneutica platonica nei secoli XIX e XX".

bibiographie

AnNAS, J., and Rowe, C., edd., Perspectives on Plato: Modern and Ancient, Cambridge, Mass., forthcoming.

BuRKE, S., Death and Return of the Author, Edinburgh University Press, 2nd edition, Edinburgh, 1998.

Grote, G., Plato and the Other Companions of Socrates, 3 vols., London, 1865.

KAHN, C., Plato and the Socratic Dialogue: The Philosophical Use of a Literary Form, Cambridge, 1996.

Nehamas, A., The Art of Living, University of California Press, Berkeley, 1998.

Stefanini, L., Platone, 2 vols. Padova, 1949, (seconda edizione; ristampata 1991).

Tigerstedt, E.N., Decline and Fall of the Neoplatonic Interpretation of Plato. An Outline and Some Observations, Commentationes Humanarum Litterarum 52, Helsinki, 1974.

Tigerstedt, E.N., Interpreting Plato, Acta Univ. Stockholmiensis, Stockholm Studies in History of Literature 17, Stockholm, 1977.

Vlastos G., Socrates: Ironist and Moral Philosopher, Cambridge, 1991. 